\title{
Proyecto de construcción de una barda escolar: Un dispositivo didáctico interinstitucional para Telesecundaria
}

\author{
Arturo Ruíz-Rojas, Instituto Politécnico Nacional (México) \\ Avenilde Romo-Vázquez, Instituto Politécnico Nacional (México) \\ Armando Solares-Rojas, Centro de Investigación y de Estudios Avanzados del \\ Instituto Politénico Nacional (México)
}

\begin{abstract}
Proyecto de construcción de una barda escolar: Un dispositivo didáctico interinstitucional para Telesecundaria

\section{Resumen}

Presentamos una investigación centrada en el diseño de un Recorrido de Estudio e Investigación (REI), enmarcada en el paradigma del Cuestionamiento del mundo (Chevallard, 2013). Exponemos el trabajo realizado en una escuela telesecundaria de una comunidad rural de México y usamos la noción de inter-institucionalidad para estudiar un problema significativo para la comunidad y el entorno social de la escuela: la construcción de la barda perimetral del edificio escolar. Este problema y su análisis constituyeron elementos clave del diseño metodológico. A través del REI, los estudiantes y la maestra lograron posicionarse y enfrentar la problemática real, desde la escuela. Además, cubrieron contenidos del programa de estudios y recurrieron a un modelo de proporcionalidad directa para abordar tareas tales como evaluar presupuestos, hacer entrevistas, investigar salarios, cantidades, calidades y resistencias de los materiales. Concluimos sobre la necesidad de generar diseños inter-institucionales enmarcados, por ejemplo, en la propuesta teórico-metodológica aquí presentada.
\end{abstract}

Palabras clave. Telesecundaria; Cuestionamiento del mundo; inter-institucionalidad; albañilería; proporcionalidad.

School building perimeter wall construction project: An interinstitutional didactic device for Telesecundaria

\section{Abstract}

Based on the perspective of "Questioning the world" (Chevallard, 2013), our research focused on designing a Study and Research Path (SRP). We present the work carried out in a 'telesecundaria' school from a rural community in Mexico and used the notion of interinstitutional relationship to study a relevant problem for the community and the school social context: the construction of the school building perimeter wall. This problem and its analysis were central in the methodological design. Through the proposed $S R P$, students and teacher managed to position themselves and face the real problem, from the school. In addition, they covered a significant amount of the syllabus contents and used a direct proportionality model to address some of the derived tasks such as evaluating budgets, making interviews, investigating salaries, quantities, qualities and resistance of the materials. We conclude about the need to generate interinstitutional designs framed, for instance, in the theoretical-methodological proposal here presented.

Keywords. 'Telesecundaria'; Questioning the world; interinstitutionality; masonry; proportionality.

\section{Introducción}

En este artículo presentamos una investigación centrada en el diseño de un Recorrido de Estudio e Investigación (REI) y enmarcada en el paradigma del Cuestionamiento del mundo (Chevallard, 2013). La investigación se desarrolló en una escuela telesecundaria de una comunidad rural del estado de Morelos, México, en la cual las familias tienen una intervención dinámica en las actividades y la vida escolar. La escuela se construyó gracias a las familias pues anteriormente compartía el edificio con otro plantel en el turno vespertino. En el momento del estudio esta telesecundaria 
funcionaba, pero aún no contaba con toda la infraestructura necesaria; entre otras cosas, faltaba una barda perimetral para delimitar el terreno de la escuela. Esta problemática abrió la oportunidad de diseñar e implementar un REI, cuyo análisis constituye el centro de este artículo. En particular, se destaca cómo el dispositivo didáctico del REI posibilita una actividad interinstitucional, al relacionar saberes de la albañilería y saberes matemáticos escolares de proporcionalidad para abordar una cuestión de interés para la comunidad. Se sostiene y evidencia que la propuesta teórico-metodológica de diseño e implementación del REI constituye una opción interinstitucional para relacionar saberes de diferente índole y una alternativa a la exigencia pedagógica de la "transversalidad" de los contenidos pedida por el plan de estudios de telesecundaria (SEP, 2017).

El artículo consta de cinco secciones. En la primera se presenta el subsistema educativo de Telesecundaria y la problemática asociada a la exigencia pedagógica de la transversalidad de contenidos. En la segunda, se exponen los elementos de la Teoría Antropológica de lo Didáctico que enmarcan esta investigación. En la tercera, se muestra la metodología, incluyendo el diseño del REI. En la cuarta sección se revelan los resultados del estudio, enfocados en la implementación del REI en un grupo de Telesecundaria. Finalmente, se presentan algunas reflexiones generales.

\section{Telesecundaria: Modalidad clave en la enseñanza mexicana}

En México, la modalidad educativa de Telesecundaria surgió a mediados de la década de los 60 para atender las necesidades educativas de pequeños asentamientos rurales, alejados de centros urbanos; lugares en los que la relativa baja cantidad de alumnos no hacía costeable establecer las escuelas secundarias públicas de la modalidad regular (Carvajal, 2006; Calixto y Rebollar, 2008). Originalmente, en las telesecundarias las clases eran impartidas por "telemaestros", mediante transmisiones de programas de televisión en vivo. Estas clases televisadas se cambiaron por clases grabadas, con alumnos y maestros como protagonistas que seguían un guion ficticio previamente diseñado. En las aulas reales, el maestro a cargo del grupo organizaba las actividades propuestas en la clase televisada, vigilaba el orden, la puntualidad y asistencia de los estudiantes, elaboraba informes y documentación escolar, y aplicaba evaluaciones. Luego se incorporaron libros de texto impresos como apoyo a las clases grabadas (Carvajal, 2006; Jiménez, Martínez y García, 2010). Desde su origen, un solo maestro se ha encargado de enseñar todas las asignaturas a los grupos de estudiantes. La modalidad de Telesecundaria ha venido creciendo en el sistema educativo mexicano, ampliando su cobertura en poblaciones rurales y atendiendo poblaciones urbanas en condiciones de marginalidad. Los Indicadores del Sistema Educativo Nacional (INEE, 2016) muestran que las telesecundarias son el $48.2 \%$ de las escuelas de secundaria del país (18.592 escuelas), atienden a $21 \%$ de la matrícula total de secundaria (1.433.818 alumnos) y el $17.7 \%$ de los maestros de educación pública labora en ellas (72.258 docentes). A pesar del crecimiento y cobertura, sus condiciones y formas de trabajo han sido poco estudiadas. Entre los temas pendientes de estudio están las maneras en que los maestros de Telesecundaria articulan las exigencias del plan de estudios con las condiciones específicas del trabajo en el salón de clases (Ávila et al., 2013).

Un objetivo del programa de estudios oficial de México (SEP, 2017) consiste en la transversalidad de contenidos que requiere vincular contenidos de asignaturas del currículum, como matemáticas, lengua, física, biología, química, historia, geografía, inglés, educación física, etcétera. Los profesores deben relacionar "conocimientos o habilidades que se hacen presentes en distintos ámbitos y momentos del currículo, como la lectura, la escritura, o el cálculo" (SEP, 2017, p. 672). Esta exigencia del programa de 
estudios se puede considerar un problema docente (Gascón, 2011) cuya solución depende de la labor delegada a los profesores de construir conocimiento suficiente en las asignaturas para establecer esta transversalidad de contenidos, con coherencia epistemológica y didáctica. En este artículo se sostiene que transformar este problema docente en un problema de investigación, implica considerar la dimensión epistemológica de las relaciones entre saberes de diferente índole. Es decir, se necesita reconocer y analizar los modelos epistemológicos que sustentan la interdisciplinariedad, la transdisciplinariedad o la co-disciplinariedad de los saberes implicados. En Takeuchi et al. (2020), "la interdisciplinariedad se refiere a la amalgama entre dos o más disciplinas, mientras que la transdisciplinariedad va más allá de la amalgama; la relación entre disciplinas no es aditiva, sino reflexiva y emergente" (traducción propia, p. 11). Para Sriraman y Freiman (2011), la interdisciplinariedad combina múltiples disciplinas académicas en una sola actividad, trascendiendo los límites disciplinarios tradicionales. Por su parte, Chevallard (2013) señala que la co-disciplinariedad reúne herramientas de diferentes disciplinas, como ilustra el paradigma del 'Cuestionamiento del Mundo', caracterizado por el estudio de cuestiones abiertas e investigables $Q$ :

La mayoría de las cuestiones generales $Q$ implican el uso de obras $O$ pertenecientes a diferentes ramas del conocimiento, por lo que el estudio de $Q$ está condenado a ser una actividad co-disciplinar, reuniendo herramientas de diferentes "disciplinas" para una causa común. (Chevallard, 2013, p. 174)

Las anteriores perspectivas se centran en las relaciones entre saberes disciplinares. Sin embargo, hay otros tipos de saberes, por ejemplo, los de prácticas comunitarias u oficios, cuya validación reposa básicamente en la experiencia y en la práctica continua; es decir, están sustentados por una base epistemológica distinta a la disciplinar. Desde la Teoría Antropológica de lo Didáctico los saberes disciplinares y no-disciplinares pueden ser vistos como producidos en distintas instituciones. Así, una actividad interinstitucional o mixta puede definirse como la que relaciona saberes de diferentes instituciones.

Nuestro objetivo es diseñar un dispositivo didáctico que posibilite una actividad interinstitucional, evidenciando elementos epistemológicos que sustentan las relaciones entre los saberes en juego y agregando una dimensión social al relacionar esta actividad con las necesidades de la escuela y de la comunidad donde se ubica la escuela en que trabajamos. Nos preguntamos qué relaciones se pueden establecer entre saberes de diferente índole mediante el diseño e implementación de un Recorrido de Estudio y de Investigación, considerado como dispositivo didáctico interinstitucional.

\section{Elementos de la Teoría Antropológica de lo Didáctico (TAD)}

La TAD ofrece un modelo epistemológico para el estudio de la actividad humana en su dimensión institucional. Dos nociones son clave: institución y praxeología.

Las instituciones, es decir, organizaciones sociales estables, enmarcan las actividades humanas y simultáneamente las hacen posibles por los recursos que estas instituciones ponen a disposición de sus sujetos. Estos recursos materiales e intelectuales han sido producidos por comunidades, a lo largo de procesos de enfrentamiento a situaciones problemáticas, para resolverlas con regularidad y eficacia (Castela y Romo, 2011, p. 85).

Así, la Telesecundaria, la enseñanza de las disciplinas, las disciplinas, los oficios y la familia son instituciones. La praxeología $[T, \tau, \theta, \Theta]$ es la una unidad mínima de análisis de la actividad humana. Sus cuatro componentes son: tipo de tareas $T$, técnica $\tau$, tecnología $\theta$ y teoría $\Theta$. La tarea es lo que se hace, la técnica es la manera en que se hace, la tecnología corresponde a discursos que producen, justifican y explican la técnica, la 
teoría corresponde a discursos más generales que producen, justifican y explican las tecnologías (Chevallard, 1999).

\subsection{Multilocalización y circulación de praxeologías entre instituciones}

Contrariamente al enfoque de la transversalidad de contenidos, en la TAD se asume una multilocalización y circulación de praxeologías entre instituciones (disciplinares, prácticas, artesanales, laborales, escolares), asociadas a procesos de transposición.

Constantemente en una institución $I$, nuevas praxeologías son vistas, por al menos una parte de actores de $I$ como necesarias para un mejor funcionamiento de $I$. Estas praxeologías deberán en consecuencia ser producidas o, aún más a menudo, reproducidas en la medida en la que existen en otra institución $I$ ' -a partir de la que se puede proponer "importarlas" a $I$. Las condiciones impuestas por la ecología de $I$ hacen entonces que la praxeología deseada no se pueda reproducir igual pues sufrirá, en esta "transferencia", diversas modificaciones adaptativas: se hablará no de transferencia, sino de transposición de $I$ ' a $I$.

Los procesos de transposición institucional no producen necesariamente versiones degradadas [...] de las organizaciones praxeológicas. [...] En todos los casos, la transposición enriquece el mundo de las praxeologías socialmente disponibles -en la medida en que ella crea una praxeología adaptada a condiciones institucionales inéditas. (Chevallard, 1999, p. 231, traducción propia).

Por tanto, diseñar una actividad didáctica interinstitucional requiere la transposición de praxeologías de otras instituciones, generando una nueva epistemología que es resultado de la recomposición de las epistemologías de las instituciones origen y destino.

\subsection{El modelo praxeológico extendido}

El modelo praxeológico extendido surgió para dar cuenta de actividades determinadas por relaciones entre saberes matemáticos e ingenieriles (Castela y Romo, 2011). Uno de sus refinamientos (figura 1) modela los procesos transpositivos relacionados con la importación de praxeologías $\left[T, \tau, \theta^{r}, \Theta\right]$ producidas en instituciones de investigación $\operatorname{Ir}$ (e.g., disciplinas) a instituciones prácticas Ip (e.g., escuelas, oficios, lugares de trabajo) con fines pragmáticos (Chaachoua, Bessot, Romo-Vázquez y Castela, 2019). El primer asterisco $I_{r}^{*}$ expresa que cada componente de la praxeología $\left[T, \tau, \theta^{r}, \Theta\right]$ puede ser objeto de transposición con relación a las epistemologías de $\operatorname{Ir}$ e Ip. La forma de hacer intervenir estas epistemologías es multifactorial, e incluye los objetivos de la transposición y la relación entre las epistemologías de Ir e Ip, entre otros. El logos $\left[\theta^{p}, \Theta^{p}\right]$ está fundamentado experimentalmente.

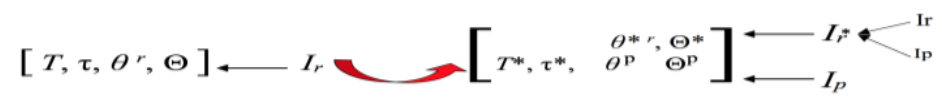

Figura 1. Modelo de procesos transpositivos de Ir a Ip (Fuente: Chaachoua, et al., 2019, p. 57)

Si con el objetivo de concebir un diseño didáctico se importa una praxeología de una institución $I x$ y se relaciona con una praxeología de una institución de enseñanza disciplinar $I e$, se obtiene una praxeología interinstitucional conformada por elementos praxeológicos de dos instituciones $I x$ e Ie. Su validez epistemológica reposa en un logos con elementos de $I x$ e $I e$, los cuales son reconocidos en el análisis de estas instituciones mediante obras, tratados, discursos de sujetos, etc. La relación entre los elementos de la praxeología interinstitucional puede ser aditiva, 'una reunión', una amalgama, 'una combinación', o una relación reflexiva y emergente, 'una recomposición'. Lo que se corresponde, en el caso de las disciplinas, con la naturaleza co-disciplinar, interdisciplinar o transdisciplinar, respectivamente. 
En el caso específico de esta investigación, se consideran dos instituciones para determinar la praxeología interinstitucional del diseño didáctico: enseñanza de las matemáticas $E(M)$ en telesecundaria y la albañilería $A$. Así, el modelo de procesos transpositivos correspondiente puede expresarse de la siguiente manera:

$$
\left.\left[T^{a}, \tau^{a}, \theta^{a} \theta^{m} \Theta^{a}\right]^{m}\right]_{\leftarrow E(M)}
$$

Con este modelo se pueden establecer distintas relaciones entre los componentes de las praxeologías de $E(M)$ y de $A$.

\subsection{El cuestionamiento del mundo un paradigma escolar}

El paradigma escolar del 'Cuestionamiento del mundo' se caracteriza por el estudio de cuestiones a través de Recorridos de Estudio y de Investigación (REI) (Chevallard, 2013). Los REI constituyen dispositivos didácticos centrados en el estudio de cuestiones abiertas e investigables $Q$. En estos dispositivos, el conjunto de estudiantes $X$ y el conjunto de los guías o profesores $Y$ emprenden procesos de investigación para determinar una respuesta llamada $R^{\vee}$, no final, pero "significativa" en relación a cierta institución $I$. El proceso de búsqueda se inicia, regularmente, a partir de ciertas cuestiones derivadas de $Q$, lo que lleva al estudio de respuestas existentes $R^{\star}$ en obras $O$ -documentos, libros, páginas web, consulta a expertos, artículos de investigación, etc.y de nuevas cuestiones. Este proceso se representa con el esquema herbartiano:

$$
\left[\mathrm{S}(\mathrm{X} ; \mathrm{Y} ; \mathrm{Q}) \rightarrow\left\{R_{1}^{\circ}, R_{2}^{\circ}, R_{3}^{\circ}, \cdots, R_{n}^{\circ}, Q_{n+1}, \cdots Q_{m}, O_{m+1}, \cdots O_{p}\right\}\right] \Rightarrow \mathrm{R}^{\bullet}
$$

La $S$ representa el sistema didáctico conformado por estudiantes y profesores que estudian la cuestión $Q$. El medio que permite la elaboración de $R^{\vee}$ está conformado por las subcuestiones, las obras estudiadas y las respuestas existentes encontradas, y por las relaciones entre estos elementos. El análisis de los REI se hace mediante dialécticas que evidencian el proceso de estudio de la cuestión $Q$. Aquí se consideran dos dialécticas: la de las cuestiones y respuestas, y la de los media-medios. La dialéctica de las cuestiones y respuestas se evidencia al identificar las $O$ consultadas y las respuestas $R^{\star}$ consideradas para producir $R^{\vee}$. La dialéctica de los media-medios consiste en el saber construido al que se considera como producto de una conjetura y que, como tal, debe ponerse a prueba (Costa, Arlego y Otero, 2015). Los media son las fuentes de información externas dirigidas a cierto sector de la población, por ejemplo, los libros de texto, las clases de un profesor, las páginas web, los artículos de investigación, etc. Los medios del estudio se conforman de los elementos tomados de los media que posibilitan la elaboración de respuestas provisionales hasta construir la respuesta $R^{\vee}$. La determinación de la cuestión $Q$, origen del REI, resulta crucial para la construcción de praxeologías en el aula.

\section{El estudio}

El diseño y análisis de la implementación del REI 'Construcción de una barda perimetral' tomó una metodología cualitativa a modo de estudio de caso (Stake, 2010).

\subsection{Diseño del REI 'Proyecto: Construcción de una barda perimetral'}

Siguiendo el método de diseño de actividades didácticas no unidisciplinares, enmarcado en la TAD, se propusieron tres fases para diseñar el REI: 1) análisis del contexto de la telesecundaria "Ing. César Uscanga Uscanga" e identificación de $Q_{0}, 2$ ) análisis y formulación de una praxeología interinstitucional y de la enseñanza de las matemáticas en telesecundaria $E(M)$ y 3) 'Proyecto: Construcción de una barda perimetral', que se establece sin que llegue a materializarse. 


\section{1) Contexto donde se ubica la escuela telesecundaria "Ing. César Uscanga Uscanga" e identificación de $Q_{0}$}

En esta fase los investigadores identificaron características del contexto social del poblado al que pertenece la escuela, así como problemáticas de la comunidad escolar de la telesecundaria "Ing. César Uscanga Uscanga" en el momento del estudio. En particular, distinguieron la necesidad de construir una barda que bordeara y resguardara el edificio escolar. El director y las familias de la escuela solicitaron la construcción de esta barda perimetral ante el ayuntamiento municipal y las autoridades educativas estatales, pero las autoridades rechazaron la solicitud argumentando que el costo de la obra sería muy alto, y que no se disponía de recursos suficientes. Sin embargo, las autoridades municipales dijeron que el costo de la construcción rebasaría los 1.5 millones de pesos, mientras que las autoridades estatales lo estimaron en 5 millones de pesos. Las diferencias en estos presupuestos resultaron sorpresivas para los padres de familia de la escuela, muchos de quienes se dedican a trabajar en la construcción como albañiles. Los padres de familia aseguraban que el costo de la obra era menor a un millón de pesos. Esta situación generó una discusión importante en la comunidad escolar.

Después de gestiones, las autoridades municipales se reunieron nuevamente con el director y las familias para elaborar otro presupuesto, más detallado, que permitiera tener un referente sobre el costo total de esta obra, así como negociar el apoyo de las autoridades estatales y federales. En la presente investigación se identifica una cuestión generatriz relevante desde el punto de vista de los conocimientos matemáticos escolares involucrados y desde la problemática de la comunidad escolar y municipal.

$Q_{0}$ : ¿Cómo generarlevaluar el diseño de una barda perimetral y su presupuesto, para una escuela Telesecundaria?

Para el estudio de $Q_{0}$ consideramos dos instituciones: la albañilería $A$ (identificada en el análisis precedente) y la enseñanza de las matemáticas $E(M)$ en Telesecundaria. La primera a través del análisis de la forma en que los albañiles generan un presupuesto; la segunda, en lo relativo al estudio de los modelos matemáticos escolares susceptibles de aparecer al contestar $Q_{0}$. Así, se estableció la naturaleza interinstitucional de la praxeología asociada al estudio de $Q_{0}$ :

$$
\left[T^{a}, \tau^{a}, \theta^{a} \theta^{m} \Theta^{a}\right]_{\leftarrow E(M)}{ }^{A}
$$

Donde el bloque práctico-técnico proviene de la albañilería $A$, mientras que el bloque tecnológico-teórico está compuesto por las tecnologías de la albañilería y de la enseñanza de las matemáticas, como se detalla a continuación.

\section{2) Análisis y formulación de una praxeología interinstitucional: Proyección de la construcción de una barda perimetral}

El análisis praxeológico de la cuestión generatriz $Q_{0}$ permitió estudiar cómo en la albañilería se hace la planeación de la construcción de una barda. Se identificaron criterios que subyacen a la elección de los materiales para la barda y maneras para calcular las cantidades y costos de materiales, de la mano de obra, e incluso del tiempo para la construcción. Este análisis permitió evidenciar modelos de proporcionalidad que funcionan como elementos tecnológicos matemáticos de la albañilería. A partir de este análisis, se reconstruye la siguiente praxeología y el paso tres de la técnica reconstruida, ya que resulta crucial para formular la praxeología interinstitucional.

Tipo de tarea $\boldsymbol{T}$ : Elaborar el diseño y presupuesto de los materiales que permitirán construir una barda perimetral. 
Tarea $\boldsymbol{t}^{\boldsymbol{a}}$ : Elaborar el diseño y presupuesto de los materiales que permitirán construir una barda perimetral para una telesecundaria.

Técnica de la albañilería reconstruida $\tau^{a}$ : Las entrevistas de un investigador a tres albañiles permitieron identificar una técnica de elaboración del diseño y presupuesto para la construcción de la barda de la Telesecundaria que engloba un gran número de pasos y subtareas, no siempre ordenados ni bien estructurados. A continuación, se presenta la reconstrucción de esta técnica considerando principalmente la entrevista a don Pedro, organizada según diferentes subtareas de esta:

1) Elegir un diseño. Los albañiles indicaron que, a partir de su experiencia, era necesario comenzar decidiendo el tipo de barda a construir. Según ellos, el diseño tradicional de una barda de escuela consiste en muros de tabique de aproximadamente 2 metros de altura y 3 metros de longitud. Al parecer, para los albañiles no es relevante la anchura que puede tener la barda; esto quizás se deba a las características de los materiales con los que generalmente se construyen estas bardas (tabiques y blocks, como se señala más adelante). Para dar soporte y resistencia a la barda, se construyen castillos -entre muro y muro- y una cadena que une todos los muros (Figura 2).

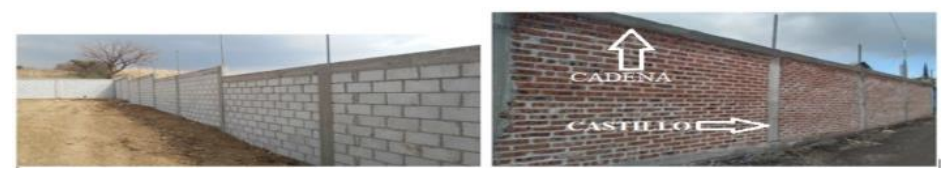

Figura 2. Bardas escolares en las que se aprecian cadenas y castillos

2) Determinar los materiales. "Se utilizaría piedra, varilla, alambre quemado, anillos, madera para cimbra, mortero, cemento, grava, arena. Para los muros, si la quieren de block, puede ser block (tipo de tabiques prefabricados) o tabique rojo recocido", señaló el maestro albañil don Pedro. Para elegir los materiales es necesario considerar durabilidad y resistencia, así como el presupuesto disponible, según él.

3) Calcular la cantidad necesaria de materiales. De acuerdo con los albañiles, en esta subtarea se define tanto la cantidad de castillos, cadenas de trabes, cimientos, etc., como la cantidad de tabiques, cemento y grava que se utilizará.

Para analizar la técnica de don Pedro para calcular los materiales, se le propuso en la entrevista realizar la subtarea $t 1^{a}$ : Determinar la cantidad de millares de tabiques necesarios para construir 100 metros de longitud de la barda. Para resolver $t_{1}{ }^{a}$, la técnica de don Pedro se basó en los cálculos por muro, la sección de barda que queda entre dos castillos (Figura 2), como se señala en el siguiente episodio.

Entrevistador: Y por ejemplo para hacer 100 metros de barda, ¿cuántos millares le calcula de tabique?, ¿cómo sé cuántos millares se ocupan?

Don Pedro: Híjole, pues sería cuestión de hacer la cuenta, pero los castillos... la separación de muro va de 3 metros de castillo a castillo [ver figura 2]. Ora póngale, serían... un millar de tabique aproximadamente desarrolla entre 18 y 20 metros cuadrados, [...] entonces estaríamos hablando que cada muro se llevaría... 2 [se refiere a 2 metros de altura] por 3 [3 metros de largo de cada muro], serían 6 metros [cuadrados] de tabique por cada muro. Ahora dele por $100 . .$. Se puede decir que en 4 muros... en 3 muros... Sería un muro... en 9 metros lineales sería lo de un millar. Se llevaría un millar de tabique en 9 metros. Ahora por $100 \ldots$

Para resolver $t_{1}{ }^{a}$ don Pedro no utiliza la regla de tres, técnica tradicionalmente estudiada en la escuela en México; volveremos sobre este uso escolar más adelante. Como se 
aprecia, don Pedro partió de conocimientos y prácticas de cálculo instauradas en la albañilería. Él sabía que: (A) El largo del muro de un castillo a otro castillo, o "separación de muro" como él lo llama, es de 3 metros. Esta es una medida que suele usarse en la albañilería. (B) La altura usual de una barda es de 2 metros. (C) Un millar de tabique alcanza ("desarrolla", dice) para entre 18 y 20 metros cuadrados de barda.

Estos conocimientos se han identificado también en otras investigaciones sobre conocimientos matemáticos involucrados en la albañilería (Fioriti, 1999; Moreira y Pardal, 2012; Padilla, 2015; Giménez, 2018). A partir de ellos, don Pedro calculó mediante aproximaciones y relaciones de proporcionalidad la cantidad de tabiques para construir la barda. Comenzó encontrando la cantidad de metros cuadrados de un muro. Para ello, usó los conocimientos A y B presentados más arriba y multiplicó 2 (cantidad de metros de la altura de barda) por 3 (cantidad de metros del largo del muro), con lo que obtuvo 6 metros cuadrados por muro. Representamos el resultado mediante la razón unitaria $\mathrm{R}_{1}=\left(1\right.$ muro, $\left.6 \mathrm{~m}^{2}\right)$. Luego, estableció una relación entre las cantidades involucradas: los millares de tabique, los muros, los metros cuadrados y los metros de largo de la barda. Desde el punto de vista de las matemáticas escolares, se trata de una relación de proporcionalidad múltiple (Block, Mendoza y Ramírez, 2010) que involucra cuatro cantidades. Para reconstruir la técnica correspondiente, se puede considerar que don Pedro tomó en cuenta tres relaciones "intermedias":

- La relación entre muros y metros cuadrados correspondientes a su superficie. Para establecerla, encontró que a 4 muros corresponden 24 metros cuadrados, y a 3 muros corresponden 18 metros cuadrados. Probablemente, obtuvo estas cantidades a partir de $\mathrm{R}_{1}=\left(1\right.$ muro, $\left.6 \mathrm{~m}^{2}\right)$.

- La relación entre un millar de tabiques, los muros y los metros cuadrados correspondientes. Para establecerla pudo combinar $\mathrm{R}_{1}$ y la razón $\mathrm{R}_{2}=(1$ millar, alcanza para 18 a $20 \mathrm{~m}^{2}$ ), dada por el conocimiento C. Al final, estableció la razón múltiple $\mathrm{R}_{3}=\left(1\right.$ millar, $18 \mathrm{~m}^{2}, 3$ muros $)$, al elegir la cota inferior de $\mathrm{R}_{2}$.

- La relación entre las cuatro cantidades. Pudo obtenerla al usar el conocimiento del inciso A, que puede representarse con la razón unitaria $\mathrm{R}_{4}=(1$ muro, $3 \mathrm{~m})$ y combinarla con las relaciones anteriores. La relación de proporcionalidad múltiple correspondiente está dada por la razón $\mathrm{R}_{5}=\left(1\right.$ millar de tabiques, $18 \mathrm{~m}^{2}$ de superficie de barda, 3 muros, 9 metros de largo de barda).

Aunque en la entrevista don Pedro no dio la respuesta ni dijo cómo calcularía la cantidad de tabiques correspondiente a 100 metros, una posibilidad es proceder nuevamente por aproximaciones: considerando el producto 9 x $11=99$, como una aproximación por debajo a 100; y, finalmente, respondiendo que se necesitarían 11 millares de tabiques. En Padilla (2015) esta manera de proceder mediante aproximaciones sistemáticas es identificada como una técnica socialmente compartida en la albañilería.

\section{Tecnología $\theta^{a}$}

De acuerdo con lo que indicó don Pedro en la entrevista, los elementos tecnológicos de la albañilería para la selección de material se refieren a su calidad y resistencia. Por ejemplo, considerar el tipo de suelo es clave para la elección de materiales, dado que a partir de esto se determina el tipo de cimentación: cuando el piso es fuerte, firme, se hace una cimentación de 80 o $90 \mathrm{~cm}$ [de profundidad]. Cuando es blando... igual un metro, nada más que hay meter unas zapatas en cada castillo, dice don Pedro. Asimismo, señaló que los costos de la construcción de la barda perimetral podrían disminuir sin descuidar los aspectos centrales que dan soporte a la estructura. Por ejemplo, en los castillos se podrían usar varillas de $3 / 8$ de pulgada, en lugar de 1/2 pulgada. Además, 
dijo que, si bien el tabique rojo es más resistente que el block, para una barda se puede elegir el block por ser más económico sin perder resistencia.

En cuanto a la técnica para determinar la cantidad de tabiques para la barda de 100 m (la subtarea $t_{1}^{a}$ ), don Pedro no utilizó las técnicas (escolares) tradicionales de la proporcionalidad escolar, como se muestra arriba; recurrió a técnicas y conocimientos con alcances y justificaciones dados por la práctica misma de la albañilería, los cuales constituyen el discurso tecnológico de la albañilería que sustenta la praxeología que el albañil usó en sus cálculos. Usó el muro para medir tanto la superficie de la barda (R1 $=\left(1\right.$ muro, $\left.\left.6 \mathrm{~m}^{2}\right)\right)$ como para calcular mediante aproximaciones la cantidad de millares de tabique necesarios $\left(\mathrm{R} 3=\left(1\right.\right.$ millar, $18 \mathrm{~m}^{2}, 3$ muros $\left.)\right)$. La cantidad de tabiques que se puede encontrar con su técnica (11 millares) corresponde a la cota máxima de tabiques que se necesita; pues de $\mathrm{R}_{2}$ se puede obtener que 9 metros de longitud de la barda es el mínimo que se puede cubrir con un millar de tabiques y 10 metros es el máximo que se puede cubrir; entonces, 99 metros es el mínimo que se puede cubrir con 11 millares de tabiques y 100 metros es el máximo que se puede cubrir con esta cantidad de tabiques. A partir de estos cálculos, se puede tener un estimado de cuántos millares se necesitan y, si se considera adecuado, elegir la cota mínima para cubrir las frecuentes pérdidas de material propias de la construcción, como las pérdidas por roturas de tabiques.

El análisis y la determinación de la tecnología $\theta^{a}$, identificada en la solución de don Pedro a la subtarea $t_{1}{ }^{a}$ permite establecer una relación con la proporcionalidad directa en su forma escolar. Es decir, la tarea de calcular la cantidad de ladrillos necesarios para la construcción de una barda puede asociarse a técnicas sustentadas por la proporcionalidad directa escolar.

\section{Tecnología $\theta^{m}$}

Se presenta un breve análisis de la tecnología matemática escolar $\theta^{m}$ que sustenta las técnicas que tradicionalmente se enseñan en la escuela para resolver la tarea $t^{a}$. Hacer explícita esta tecnología matemática escolar permitió contrastar y articular los conocimientos escolares con los conocimientos movilizados por la praxeología de la albañilería en la resolución de la cuestión generatriz $Q_{0}$ identificada en la fase 1.

Si bien el programa de estudios con el que se trabaja en telesecundaria (SEP, 2017) promueve el estudio de distintos problemas y técnicas clásicos de la proporcionalidad ${ }^{1}$, en general en las clases regulares no se estudian problemas "complejos", como la tarea $t^{\text {a }}$ : Elaborar el diseño y presupuesto del material para construir una barda perimetral en una Telesecundaria. Este tipo de problemas requiere la toma de decisiones y la elaboración de subtareas que permiten identificar respuestas y técnicas ya existentes en otras obras matemáticas y de otras disciplinas hasta elaborar una solución a la tarea originalmente propuesta (Parra y Otero, 2017).

La investigación ha documentado que las explicaciones tecnológicas escolares están generalmente restringidas al modelo del que provienen y no permiten resolver problemas "complejos" ni articular conocimientos con otras áreas de las matemáticas ni otras disciplinas. Bosch (1994) identificó distintas modelizaciones escolares de la proporcionalidad en las que hay saltos y rupturas que promueven la enseñanza de las matemáticas centrada en contenidos y operaciones aislados. En contraste, en la tecnología que usó don Pedro para la solución de la subtarea $t_{1}{ }^{a}$, las aproximaciones y

\footnotetext{
1 Se incluyen problemas de valor faltante, comparación de razones, proporcionalidad múltiple, composición de relaciones de proporcionalidad; por el lado de las técnicas, se estudian las razones internas, la razón externa o constante de proporcionalidad, el valor unitario, por ejemplo.
} 
justificaciones están ligadas a la actividad de la albañilería, a sus conocimientos, prácticas y restricciones, los cuales rebasan lo "completamente matemático" e incluyen conocimientos y criterios de otros ámbitos. Además de sus abundantes conocimientos sobre las relaciones matemáticas complejas involucradas, para la solución de la tarea $t^{\text {a }}$, don Pedro considera aspectos como la durabilidad y resistencia de los materiales, así como la cantidad de dinero disponible para la construcción.

Si se usa una estrategia escolar clásica, se puede plantear la solución del problema mediante la aplicación de la fórmula del área de un rectángulo (largo $\times$ ancho) y la "regla de tres", como a continuación se presenta:

La barda pedida tiene $100 m \times 2 m=200 \mathrm{~m}^{2}$ de superficie. Se sabe que 1 millar de tabiques alcanza para entre $18 \mathrm{~m}^{2}$ y $20 \mathrm{~m}^{2}$. ¿Cuántos millares se necesitan para $200 \mathrm{~m}^{2}$ ? Para resolver esta "subcuestión" se puede plantear dos veces una regla de tres:

$$
\begin{gathered}
18 \mathrm{~m}^{2}\left(20 \mathrm{~m}^{2}\right) \text { se cubren con } 1 \text { millar de tabiques } \\
200 \mathrm{~m}^{2} \text { se cubren con } x \text { millares de tabiques } \\
x=\frac{200 \mathrm{~m}^{2} \times 1 \text { millar de tabiques }}{18 \mathrm{~m}^{2}}=11.11 \text { millares de tabiques } \\
\left(x=\frac{200 \mathrm{~m}^{2} \times 1 \text { millar de tabiques }}{20 \mathrm{~m}^{2}}=10 \text { millares de tabiques }\right)
\end{gathered}
$$

Y así concluir que se requiere entre 10 y 11.11 millares de tabiques para construir la barda. Si bien esta solución usa conocimientos de la albañilería, no se usan los criterios ni las técnicas que sí usó don Pedro en la entrevista. Emergen así las preguntas: ¿cómo articular la praxeología escolar con la praxeología de los albañiles?, ¿en qué medida es pertinente trasponer a la escuela tareas, técnicas y tecnologías que permitan recuperar la riqueza de la actividad matemática realizada en la institución de los albañiles?, ¿cómo hacerlo? La noción de interinstitucionalidad da respuestas a estas preguntas.

\section{3) REI 'Proyecto: Construcción de una barda perimetral'}

El REI se propuso como una convocatoria para hacer un proyecto de construcción de una barda perimetral y se basó en las dos fases anteriores. La primera permitió identificar y transponer la cuestión generatriz $Q_{0}$ : ¿Cómo generar/evaluar el diseño de una barda perimetral y su presupuesto, para una escuela Telesecundaria? La segunda evidenció que el estudio de $Q_{0}$ requiere de una praxeología interinstitucional que involucra saberes matemáticos escolares - como los de proporcionalidad-y saberes de la albañilería de los cuales identificamos los relativos a tipos y cantidades de materiales. El REI se presentó a través de una convocatoria para participar en la elaboración del proyecto: 'Construcción de una barda perimetral' (Figura 3). En el aula hubo tres etapas: i) diseño de la barda, ii) cálculo del presupuesto para construir la barda y iii) construcción de una maqueta de la barda perimetral. Aquí se analiza la etapa 2 que permite evidenciar las relaciones entre saberes matemáticos escolares y saberes de la albañilería, así como las formas en que se relacionan en la praxeología interinstitucional.

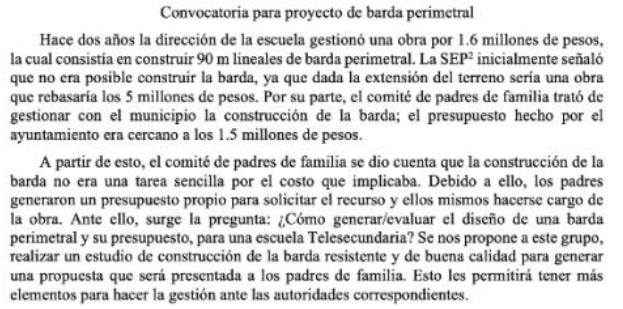

Figura 3. Convocatoria para proyecto: Construcción de una barda perimetral 


\subsection{Condiciones de implementación del REI y participantes}

La implementación del REI estuvo a cargo de la maestra titular del grupo y de un investigador, quienes previamente trabajaron la forma de implementarlo, discutiendo los objetivos y alcances de las actividades propuestas. Los estudiantes participantes fueron 24 , de entre 14 y 17 años de edad, quienes trabajaron en 3 equipos de 8 personas cada uno y como grupo durante las puestas en común. La maestra dedicó dos sesiones de una hora por semana, durante tres meses. El rol del investigador en la implementación fue de observador-participante. Cada dos semanas, asistió al aula para atender las preguntas y dudas de la maestra así como para ayudar en las definiciones de las actividades a realizarse en las sesiones subsecuentes. En ocasiones colaboró en la puesta en común para intercambiar experiencias de los estudiantes, aprovechando para realizar preguntas sobre sus producciones y motivándolos a profundizar en sus investigaciones.

\subsection{Insumos para el análisis}

Las interacciones de los estudiantes fueron videograbadas durante las clases y se recopilaron sus producciones escritas, las hojas de trabajo y los tres pequeños reportes que elaboraron en equipos, correspondientes a cada una de las tres etapas del proyecto. Las interacciones con la maestra de grupo fueron registradas en un cuaderno de notas, y fueron objeto de análisis en las reuniones que sostuvieron los tres investigadores del estudio para decidir sobre las actividades que se realizarían en las siguientes sesiones.

\subsection{Categorías de análisis de la implementación del REI}

El análisis del REI de los estudiantes se basó en la identificación de dos dialécticas: (a) la de las cuestiones y respuestas, y (b) la de los media-medios. Para ello, se analizaron las hojas de respuestas entregadas por los equipos de estudiantes, los tres reportes asociados a las tres etapas del desarrollo en el salón de clases del REI y episodios seleccionados de las clases videograbadas. Los episodios corresponden a segmentos de video que permitieron identificar las dialécticas, objeto de análisis. El 'cuestión-grama', herramienta dinámica para el análisis de los REI (Barquero, Bosch y Romo, 2018) fue utilizado para evidenciar la dialéctica de las cuestiones y respuestas. Los tres investigadores participaron en el análisis, inicialmente de forma individual y, posteriormente, de forma colectiva. Esta forma de trabajo permitió profundizar, triangular y consensuar los análisis individuales iniciales. El conjunto de los elementos metodológicos presentados posibilitó el diseño, implementación y análisis del REI.

\section{Resultados}

El REI producido por los alumnos se ilustra parcialmente mediante un "cuestióngrama" (Figura 4). El análisis de la cuestión $Q_{2}$ y de sus cuestiones derivadas $\left(Q_{2.1}, Q_{2.2}\right.$ $Q_{2.3 \ldots}$ ) constituye la base de los resultados presentados en esta sección, para evidenciar la reconstrucción de elementos de la praxeología interinstitucional en el aula.

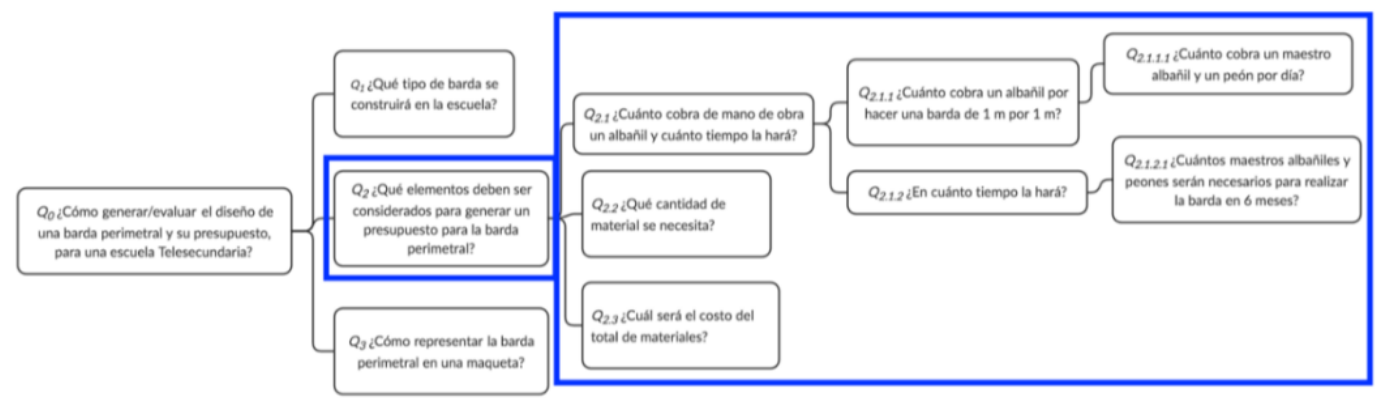


Figura 4. Cuestión-grama del REI de los alumnos

\subsection{Generar un presupuesto y el uso de un modelo proporcional}

Los alumnos y la maestra en una puesta en común decidieron realizar entrevistas para generar el presupuesto. Los alumnos entrevistaron a cuatro albañiles (Onésimo, Juan, Luis y Pancho), padres de familia y un arquitecto, e hicieron consultas a un local de venta de materiales de construcción. De acuerdo al esquema herbartiano, las consultas y las entrevistas constituyeron el estudio de obras $O$ representadas por discursos orales y escritos de expertos ${ }^{2}$, las respuestas obtenidas $R$ y las nuevas cuestiones conformaron en su conjunto el medio didáctico $M$, que permitió a los alumnos la elaboración del presupuesto, como una respuesta para $Q_{2}$. De manera general, los alumnos hicieron tres preguntas base $Q_{2.1}, Q_{2.2}$ y $Q_{2.3}$ en las entrevistas a los albañiles, quienes constituyeron medias (fuentes de información) útiles para elaborar $R^{\boldsymbol{v}}$.

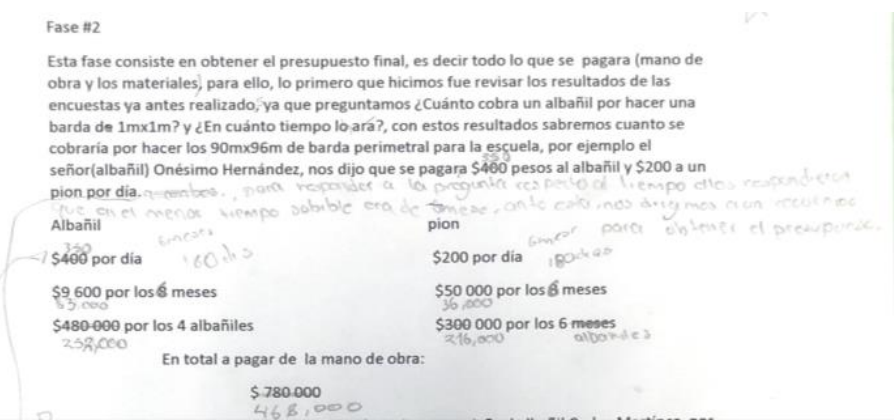

Figura 5. Extracto del reporte con cálculos sobre mano de obra de los albañiles

Los alumnos propusieron una "unidad de barda de $1 \mathrm{~m}$ x $1 \mathrm{~m}$ " $\left(Q_{2,1,1}\right)$, al igual que don Pedro, considerando solo su superficie. Los albañiles entrevistados, Juan y Onésimo, por el equipo 1, indicaron que la mano de obra se determina a partir del salario diario por el número de días de duración de la construcción de la barda. Las cuestiones $Q_{2,1,1}$ y $Q_{2,1,2.1}$ permitieron a los alumnos considerar el salario diario de los albañiles, lo que escolarmente se llama valor unitario de la relación pago-tiempo ${ }^{3}$. Onésimo indicó que hay dos tipos de albañiles: maestro albañil y peón, con salarios diarios distintos.

En respuesta a $Q_{2,1,1}$, los estudiantes obtuvieron de la entrevista de Juan, que el maestro albañil cobra $\$ 350.00$ por día y el peón $\$ 200.00$ (a diferencia de lo que Onésimo les había informado), y sobre-escribieron con lápiz estas cantidades (figura 5). Para la pregunta $Q_{2,1,2.1}$ de la entrevista de Juan obtuvieron que se requieren 4 maestros albañiles y 6 peones para realizar la barda en 6 meses. Estas dos respuestas les permitieron calcular el costo de la mano de obra. Consideraron 6 meses de 30 días (180 días en total), que multiplicados por el salario diario del maestro albañil es de $\$ 63,000$; y esto por cuatro (número total de maestros albañiles) da un costo de $\$ 252,000.00$. De la misma manera, determinaron el costo de mano de obra de los peones: $\$ 200.00$ por día, considerando 180 días, obtuvieron que el salario de un peón es de $\$ 36,000.00$; y de los seis peones es de $\$ 216,000.00$. Se puede notar que no consideraron que los domingos no se trabaja y que esos días no se pagan en la albañilería (al menos en el contexto de la comunidad donde se encuentra la escuela). Estas maneras de hacer los cálculos y de

\footnotetext{
${ }^{2}$ El arquitecto se entrevistó vía correo electrónico y sus respuestas utilizadas en las etapas 1 y 3 del REI. ${ }^{3}$ En una relación de proporcionalidad entre dos conjuntos de cantidades A y B, hay dos valores unitarios: el valor que en el conjunto B corresponde a la unidad del conjunto A, y viceversa. En la relación pagotiempo que se discute aquí los valores unitarios son la cantidad de dinero que se paga a un albañil por día (una unidad de tiempo) y la cantidad de tiempo que tiene que trabajar para que se le pague un peso (una unidad de dinero). Es este caso, el segundo valor unitario generalmente no se usa en la albañilería.
} 
elegir las cantidades y relaciones relevantes para el problema constituyen las técnicas que los alumnos van desarrollando y proponiendo a lo largo del REI.

$\begin{array}{llll}\text { Mamposteo } & \$ 220 \times \text { Metro } & 366 \text { Metros } & \$ 80,520 \\ \text { Muro } & \$ 180 \times \text { Metro } & 732 \text { Metros } & \$ 131,760 \\ \text { Cadena } & \$ 200 \times \text { Metro } & 735 \text { Metros } & \$ 147,000 \\ \text { Castillo } & \$ 666.50 \times \text { Metro } & 279 \text { Metros } & \$ 186,000\end{array}$

Figura 6. Extracto del reporte del equipo 2

Para responder $Q_{2.1}$, y $Q_{2.2}$, los equipos 2 y 3, entrevistaron cada uno a un albañil, Luis y Pancho. Con base en las respuestas obtenidas, estos dos equipos establecieron relaciones de la proporcionalidad directa entre las cantidades involucradas, aunque de forma distinta. Por ejemplo, ellos encontraron respectivamente en la entrevista y en la consulta hecha a un local de venta de materiales de construcción que el costo de mano de obra se determinaba por el "tipo de trabajo realizado" (Figura 6). Así, para ellos el tiempo en la realización no fue un factor determinante para el costo en este rubro. Los equipos 2 y 3 utilizaron un modelo de proporcionalidad directa que está determinado por la relación entre el costo de la mano de obra, el valor unitario de la mano de obra por metro y la cantidad de metros; siguiendo para cada tipo de trabajo una forma general del siguiente modo: costo de la mano de obra = valor unitario del tipo de trabajo $\times$ cantidad de metros de la obra. Nuevamente, se trata de las técnicas y las elecciones que los alumnos construyen en el desarrollo del REI en el salón de clases.

Los costos que presentan los estudiantes en la Figura 6, solo para los castillos dan un resultado "redondeado". Si bien no sabemos la razón exacta de este redondeo, sí se puede notar que en este caso el valor unitario del pago por metro es una cantidad decimal: $\$ 666.50$ (en los otros casos los valores unitarios son enteros). Siguiendo el modelo de proporcionalidad que eligieron los estudiantes, el resultado exacto para el costo de la mano de obra de los castillos es: $\$ 185,953.50$. Este redondeo puede obedecer a criterios de facilidad de manejo de cantidades enteras y múltiplos de 10.

\subsection{La dialéctica media-medios en la determinación de presupuesto}

Los tres equipos manifestaron que los materiales y cantidades fueron dados por los albañiles en la entrevista $\left(Q_{2.2}\right)$, aunque el procedimiento para calcularlos no se expuso. Las entrevistas a los albañiles que realizaron estudiantes como parte de la proyección de la construcción de una barda, y específicamente de la determinación del presupuesto para su construcción, constituyen referentes para los criterios de determinación de las cantidades y costos de los materiales, así como de las posibles técnicas de cálculo. Es también uno de los referentes que figuran en el análisis y formulación de la praxeología interinstitucional de proyección de la construcción de una barda perimetral.

Más allá de la técnica utilizada por los albañiles entrevistados (media), la técnica del valor unitario (como en el caso de la cantidad de dinero pagado por la cantidad de metros construidos) es un medio utilizado en varios momentos de manera efectiva, lo que hace suponer que los estudiantes utilizaron esta técnica para calcular también las cantidades de materiales. Asimismo, el dominio y la práctica de los conocimientos y las técnicas de la albañilería de los maestros albañiles les permitió realizar cálculos de manera muy rápida y eficiente sobre el rendimiento de diversos materiales, como arena, grava y mortero en algunas unidades de referencia, lo cual facilita el cálculo de los materiales para proporcionarlos a los alumnos.

\subsection{Técnica del valor unitario, medio escolar privilegiado}


Con respecto a $Q_{2.3}$, los equipos 1 y 2 utilizaron un modelo de proporcionalidad directa de la forma: valor unitario del material $\times$ cantidad de material $=$ costo $($ medio $)$. En cambio, el equipo 3 mostró solo el valor unitario y el costo total, omitiendo la cantidad de material. Este acercamiento a la albañilería motivado por este REI permitió reconocer elementos clave para determinar el presupuesto de la barda: los materiales y sus cantidades, costo de la mano de obra, roles de los trabajadores de la construcción y tiempo de duración de la obra. Las técnicas expuestas por los albañiles en las entrevistas parecen, sin embargo, permear de manera menos visible el trabajo de los alumnos. Esto puede deberse a que recrear las condiciones de la albañilería en la escuela es complejo y los alumnos no poseen los saberes prácticos que justifican las técnicas de la albañilería. En este sentido, cabe considerar otras cuestiones o subcuestiones que puedan integrarse al REI propuesto a fin de acercarse a saberes prácticos y a la forma en que estos pueden ser útilmente relacionados con saberes escolares, tales como: ¿cuáles son las proporciones de materiales para hacer una mezcla a base de cemento?, ¿cuáles son las proporciones de materiales para hacer una mezcla a base de mortero?, ¿qué cantidad de mezcla y de materiales se necesitan para hacer un castillo para la barda propuesta?, entre otras más que si bien no fueron explícitas, se presume fueron contempladas de manera implícita durante los diversos cálculos de materiales y costos.

\section{Reflexiones finales}

La modalidad de Telesecundaria ha cobrado gran importancia en México tanto por la cantidad de sus planteles como por el gran número de alumnos que atiende. En esta investigación, se cuestiona la exigencia pedagógica oficial de la transversalidad de contenidos, que demanda relacionar conocimientos de distintas disciplinas y momentos del currículo. Se considera que la realización de esta exigencia es sumamente compleja si se delega solo a los profesores, para completarla de manera aislada y con los pocos recursos didácticos que se pone a su disposición. Esto es debido a que los saberes disciplinares y no disciplinares se construyen bajo sistemas lógicos y normativos regidos por cada institución, por lo que su circulación entre diferentes instituciones y su difusión a través de la enseñanza están asociados a complejos procesos transpositivos, difíciles de operar en todos los diseños didácticos de la práctica diaria (Lave y Gibson, 2011; Noss, Hoyles y Pozzi, 2002; Nunes, Carraher y Schliemann, 1993).

Como una alternativa a ese enfoque pedagógico, se propone el paradigma del "Cuestionamiento del mundo", caracterizado por el estudio de cuestiones $Q$ cruciales para la comunidad. $Q$ se determinó a través del análisis y formulación de una praxeología interinstitucional que relacionó elementos de albañilería y de matemática escolar en el proyecto de construcción de una barda perimetral para una escuela. El estudio de $Q$ implicó realizar un presupuesto de construcción de la barda, los alumnos eligieron a los albañiles como medias principales, pero conformaron su medio relacionando elementos del discurso de los albañiles con la técnica escolar de "valor unitario" x "cantidad" = costo. Por tanto, la dimensión interinstitucional del REI estuvo determinada por el análisis y formulación de una praxeología de albañilería, la cual se puso en relación con las matemáticas escolares a través, entre otros elementos, de técnicas y tecnologías que involucran relaciones de proporcionalidad directa. Como lo muestra esta investigación, el desafío didáctico consiste en relacionar instituciones prácticas cuya epistemología está basada en la experiencia -como la albañilería- con instituciones de enseñanza o disciplinares. La propuesta teórico-metodológica de esta investigación constituye una vía para relacionar estas instituciones, mediante la construcción de una praxeología mixta/interinstitucional. Otros estudios de este tipo permitirán precisarla y enriquecerla. 


\section{Referencias}

Ávila, A., Carrasco, A., Gómez, A. A., Guerra, M. T., López, G. y Ramírez, J. L. (Coord.) (2013). Una década de investigación educativa en conocimientos disciplinarios en México. Matemáticas, ciencias naturales, lenguaje y lenguas extranjeras 2002-2011. México DF: ANUIES.

Barquero, B., Bosch, M. y Romo, A. (2018). Mathematical modelling in teacher education: dealing with institutional constraints. ZDM, 50(1-2), 31-43.

Block, D., Mendoza, T. y Ramírez, M. (2010). ¿Al doble le toca el doble? La enseñanza de la proporcionalidad en la educación básica. México D: SM de ediciones S.A. de C.V.

Bosch, M. (1994). La dimensión ostensiva de la actividad matemática. Trabajo de Tesis Doctoral. Universitat Autònoma de Barcelona.

Carvajal, E. (2006). Interacción en las aulas de la telesecundaria: Un acercamiento desde la enseñanza de las matemáticas. Revista Latinoamericana de Estudios Educativos, XXXVI(3-4), 129-157.

Castela, C. y Romo-Vázquez, A. (2011). Des mathématiques à l'automatique: étude des effets de transposition sur la transformée de Laplace dans la formation des ingénieurs. Recherches en Didactique des Mathématiques. 31(1), 79-130.

Calixto R. y Rebollar A. (2008). La telesecundaria ante la sociedad del conocimiento. Revista Iberoamericana de Educación, 44(7), 1-11.

Chaachoua, H., Bessot, A., Romo-Vázquez, A. y Castela, C. (2019). Developments and functionalities in the praxeological model. En M. Bosch, Y. Chevallard, F. J. García y J. Monaghan (Eds.), Working with the anthropological theory of the didactic: A comprehensive casebook (pp. 41-59). Londres, Reino Unido: Routledge.

Chevallard, Y. (1999). L'analyse des pratiques enseignantes en Théorie Anthropologique du Didactique. Recherches en Didactique des Mathématiques, 19(2), 221-266.

Chevallard, Y. (2013). Enseñar matemáticas en la sociedad de mañana: alegato a favor de un contraparadigma emergente. Journal of Research in Mathematics Education, 2(2), 161-182.

Costa, V., Arlego, M. y Otero, R. (2015). Las dialécticas en un Recorrido de Estudio e Investigación para la enseñanza del cálculo vectorial en la Universidad. Revista de Formación e Innovación Educativa Universitaria, 8(3), 146-161

Fioriti, G. (1999). Conocimiento geométrico de los obreros de la construcción: conocimiento situado versus conocimiento escolar. Trabajo de Maestría. Universitat Autònoma de Barcelona.

Gascón, J. (2011). Las tres dimensiones fundamentales de un problema didáctico. El caso del álgebra elemental. Revista Latinoamericana de Investigación En Matemática Educativa, 14(2), 203-231

Giménez, A. (2018). Prácticas donde subyacen conocimientos matemáticos en grupos de albañiles en obras pequeñas. Trabajo de Maestría. Universidad Nacional de Córdoba, Argentina.

INEE (2016). Panorama educativo de México 2015. Indicadores del Sistema Educativo Nacional. Educación Básica y Media Superior. México DF: Instituto Nacional para la Evaluación de la Educación. 
Jiménez, J., Martínez, R. y García, C. (2010). La Telesecundaria en México: Un breve recorrido histórico por sus datos y relatos. México DF: SEP.

Lave, J. y Gibson, T. (2011). Apprenticeship in critical ethnographic practice. Chicago, EEUU: University of Chicago Press

Moreira, D. y Pardal, E. (2012). Mathematics in mason's workplace. Adults Learning Mathematics: An International Journal. 7(1), 31-47.

Noss, R., Hoyles, C. y Pozzi, S. (2002) Abstraction in expertise: A study of nurses' conceptions of concentration. Journal for Research in Mathematics Education 33(3), 204-229.

Nunes, T., Schliemann, A. D. y Carraher, D. W. (1993). Street mathematics and school mathematics. Nueva York: Cambridge University Press.

Padilla, E. (2015). Conocimientos matemáticos de menores trabajadores. El caso de la proporcionalidad. Trabajo de Maestría. Universidad Pedagógica Nacional, México.

Parra, V. y Otero, M. R. (2017). Enseñanza de la matemática por recorridos de estudio e investigación: Indicadores didáctico-matemáticos de las "dialécticas". Educación Matemática, 29(3), 9-49.

Sriraman, B. y Freiman, V. (Eds.) (2011). Proceedings $3^{\text {rd }}$ International Symposium on Mathematics and Its Connections to Arts and Sciences. Montana, EEUU: IAP

SEP (2017). Aprendizajes clave para la educación integral. Plan y programas de estudio para la educación básica. México DF: SEP

Stake, R. E. (2010). Investigación con estudios de caso. Madrid: Ediciones Morata.

Takeuchi, M. A., Sengupta, P., Shanahan, M. C., Adams, J. D. y Hachem, M. (2020). Transdisciplinarity in STEM education: A critical review. Studies in Science Education, 56, 213-253.

\section{Referencias de los autores}

Arturo Ruiz-Rojas, Instituto Politécnico Nacional (México). ruiz_arturo2@hotmail.com

Avenilde Romo-Vázquez, Instituto Politécnico Nacional (México). aromov@ipn.mx Armando Solares-Rojas, Centro de Investigación y de Estudios Avanzados del Instituto Politénico Nacional (México). asolares@ cinvestav.mx

\section{School building perimeter wall construction project: An interinstitutional didactic device for Telesecundaria}

Arturo Ruíz Rojas, Instituto Politécnico Nacional, Centro de Investigación en Ciencia Aplicada y Tecnología Avanzada

Avenilde Romo Vázquez, Instituto Politécnico Nacional, Centro de Investigación en Ciencia Aplicada y Tecnología Avanzada

Armando Solares-Rojas, Departamento de Matemática Educativa, Centro de Investigación y Estudios Avanzados del IPN 
We present a research focused on a Study and Research Path design (SRP) framed into the paradigm of 'Questioning the World', which is characterized by the study of open questions (Chevallard, 2013). The process of study consists of consulting information sources, identifying existing answers, and generating derived questions. In this way it is constituted a milieu to elaborate a partial but robust answer, agreed upon by the study community, students and teacher. Thereby, mathematics is seen as knowledge that can be constructed, adapted and related to other knowledge in order to understand and modify the social environment and even the world. Our research was carried out in a rural Telesecundaria school in Mexico. This school has the particularity of very active families who participate in many school activities. At the time of the study, the school still did not have all the necessary infrastructure and lacked a perimeter fence to delimit its terrain. This situation opened the opportunity to design and implement a SRP, whose analysis constitutes the focus of this article. Based on the analysis developed, we question the official pedagogical requirement named content transversality, which demands relating knowledge from different disciplines of the curriculum. As an alternative, we propose questioning the world by means of the study of crucial $Q$ questions by the community. $Q$ came out of the analysis and formulation of an interinstitutional praxeology that related elements of masonry and school mathematics within the project of building a perimeter fence for the school. It implied calculating a budget for the fence construction for which students chose interviewing masons as the main media. They also constructed their milieu by relating elements of the masons' discourse with their school knowledge about proportional relations. Thus, the inter-institutional dimension of the REI was determined by the analysis and formulation of a masonry praxeology, which was related to school mathematics through techniques and technologies that involve direct proportionality relations. The theoreticalmethodological proposal of this research constitutes a way to relate practical institutions whose epistemology is based on experience with teaching or disciplinary institutions, through the construction of a mixed and inter-institutional praxeology. 